\title{
Sacolas oxibiodegradáveis: degradação em decorrência da condição de descarte
}

\author{
Fábio Zanella ${ }^{1}$, Robson Alves de Moraes $^{2}$, Nereide Brizola de \\ Lima $^{2}$, Ana Lúcia da Silva Lima ${ }^{1}$
}

${ }^{1}$ Instituto Federal de Santa Catarina. Campus São Lourenço do Oeste. Rodovia SC 480, S/№. Distrito de Frederico Wastner. São Lourenço do Oeste-SC, Brasil (CEP 89994-000). E-mail: fabio.zanella@ifsc.edu.br.

${ }^{2}$ Curso Técnico em Vendas do Instituto Federal de Santa Catarina (IFSC) - Campus São Lourenço do Oeste. Rodovia SC 480, S/№. Distrito de Frederico Wastner. São Lourenço do Oeste-SC. Brasil (CEP 89994-000).

Resumo. A magnitude do impacto ambiental das sacolas plásticas está estreitamente vinculada à persistência desse tipo de embalagem no ambiente. A tecnologia oxibiodegradável foi adotada no Brasil em 2007, como alternativa para minimizar o impacto ambiental das embalagens plásticas. Assim, o presente trabalho objetivou avaliar o processo de degradação de sacolas plásticas oxibiodegradáveis em diferentes condições de descarte. 0 experimento foi desempenhado no Município de São Lourenço do Oeste, Estado de Santa Catarina, entre agosto e novembro de 2017. Foram testados cinco tratamentos simulando as seguintes condições de descarte: $\mathrm{T} 1=$ a céu aberto; $\mathrm{T} 2=$ abrigado; $\mathrm{T} 3=$ na água; T4 = sob solo seco; T5 = sob solo úmido. Adotou-se a perda de massa como variável indicadora da degradação das sacolas, aos $0,30,60$ e 90 dias de tratamento. Após 90 dias, a perda de massa das sacolas não alcançou valores substanciais, sendo os maiores decréscimos observados nos tratamentos a céu aberto (T1), na água (T3) e sob solo úmido (T5), com valores de 3,4\%, $2,6 \%$ e $3,1 \%$, respectivamente. Os tratamentos T2 (abrigado) e T4 (solo seco) não expressaram decréscimos estatisticamente significativos para a massa das embalagens, comparando-se os tempos 0 e 90 dias de tratamento. Conclui-se que os descartes a céu aberto, na água e sob solo úmido foram os que mais promoveram a degradação das sacolas com embalagem oxibiodegradável, para as condições deste experimento.

Palavras-chave: Plástico oxibiodegradável; Perda de massa; Degradação; Impacto ambiental.

\begin{abstract}
Oxybiodegradable bags: degradation due to the disposal condition. The magnitude of environmental impact of plastic bags is tightly linked to the persistence in the environment of this class of packaging. Brazil has been adopted the oxobiodegradable technology in 2007 , as an alternative in order to minimize the environmental impact from plastic packaging. Thus, this research aimed to evaluate the degradation process of
\end{abstract}

Recebido:

21/12/2017

Aceito:

19/03/2018

Publicado:

$30 / 04 / 2018$

Acesso aberto

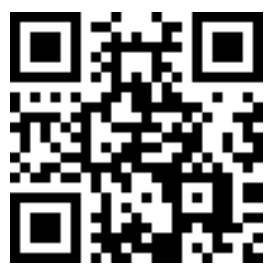

ORCID

(D) 0000-0003-4450-8239 Fábio Zanella

D 0000-0003-2473-5338 Robson Alves de Moraes

(ㄷ) 0000-0001-9800-9174 Nereide Brizola de Lima

D $0000-0002-3798-2568$ Ana Lúcia da Silva Lima 
oxobiodegradable plastic bags in different disposal conditions. The experiment was developed in Municipality of São Lourenço do Oeste, Santa Catarina State, from August to November 2017. It was tested five treatments simulating the following disposal conditions: $\mathrm{T} 1$ = open air; $\mathrm{T} 2$ = sheltered; $\mathrm{T} 3=$ water; $\mathrm{T} 4=$ dry soil; T5 $=$ wet soil. It was adopted the loss mass as indicator of bag's loss mass after 0, 30, 60 and 90 days. After 90 days, the loss mass of the bags did not reached substantial rates, with largest decreases observed under open air (T1), water (T3) and wet soil (T5), showing respective values of $3.4 \%, 2.6 \%$ and $3.1 \%$. Treatments T2 (sheltered) and T4 (dry soil) expressed no remarkable decreases to the packaging plastic by comparing 0 and 90 days of disposal. Under the conditions of the experiment, it may conclude that the disposal on open air, water and wet soil were those that promoted the larger degradation of the oxobiodegradable bags.

Keywords: Oxobiodegradable plastic; Mass loss; Degradation, Environmental impact.

\section{Introdução}

A Lei no 12.305/2010 (Brasil, 2010), que institui a Política Nacional de Resíduos Sólidos (PNRS) que, além de determinar o fim dos lixões, hierarquizou a gestão e o gerenciamento de resíduos sólidos, não geração, redução, reutilização, reciclagem, tratamento e disposição final ambientalmente adequada dos rejeitos, além da responsabilidade compartilhada dos setores privado e público (Oliveira e Galvão Junior, 2016).

Nos últimos anos, a geração de resíduos sólidos tem aumentado a uma razão cerca de cinco vezes maior do que o crescimento populacional. De acordo com Pereira e Curi (2013), o crescimento econômico do país impulsionou o poder de compra da população, a qual passou a consumir excessivamente e a gerar "lixo" em proporções alarmantes, passando este resíduo a poluir cada vez mais o meio ambiente.

0 uso de embalagens plásticas é uma das principais vias de geração de resíduos sólidos no ambiente. Historicamente, a inserção e crescimento das sacolas plásticas no mercado, substituindo as tradicionais embalagens de papel, teve início na década de 1970.
Entre as vantagens do plástico pode-se destacar sua leveza, baixo custo, selabilidade sob calor, transparência, flexibilidade, assepsia e capacidade de suportar peso sem romper-se (Santos et al., 2012), por isto sendo popularizada como sacolinhas.

As sacolinhas têm alto custo ambiental, uma vez que são produzidas a partir de petróleo ou gás natural (recursos naturais não-renováveis) e, depois de usadas, em geral por uma única vez, costumam ser descartadas de maneira incorreta, levando cerca de 450 anos para se decompor. Neste tempo, aumentam a poluição, entopem bueiros impedindo o escoamento das águas das chuvas ou vão parar em matas, rios e oceanos, onde acabam engolidas por animais que morrem sufocados ou presos nelas (Ecodebate, 2016).

0 amplo uso das sacolinhas plásticas deve-se ao fato de que estas são recebidas gratuitamente nas compras do mercado, farmácias, lojas de conveniência e entre outros locais ou adquiridas em estabelecimentos comerciais (Miranda e Seo, 2015).

Dentre as inúmeras tecnologias de produção de filmes plásticos para a produção de embalagens, destacam-se os plásticos oxibiodegradáveis. Os quais 
possuem fabricação diferenciada, apresentam aditivos pró-degradantes que aceleram o processo de degradação, ou seja, quando estes aditivos são incluídos no processo de manufatura normal, eles causam a decomposição do plástico em água, dióxido de carbono e uma pequena quantidade de biomassa, através de uma combinação qualquer de luz, calor e estresse (Polifilme Embalagens, 2013). No entanto, há estudos que não corroboram os dados de acelaração da degradação de filmes plásticos promovida pelos aditivos oxibiodegradantes. Follmann et al. (2017), por exemplo, concluíram não haver diferença significativa entre a decomposição de sacolas oxibiodegradáveis e as convencionais.

o Brasil adotou, em 2007, a tecnologia oxibiodegradável como alternativa para minimizar o impacto ambiental das embalagens plásticas convencionais. Entretanto, há certa contradição quanto à biodegrabilidade dos plásticos aditivados para a aceleração da degradação no ambiente.

Fiore et al. (2014) apontam as sacolas oxibiodegradáveis como uma medida ambientalmente incorreta, já que elas se transformam em pó, poluindo ainda mais o meio, além disso, quando isto ocorre, são necessários catalisadores. Os aditivos empregados na fabricação das sacolas oxibiodegradáveis podem ser prejudiciais ao meio ambiente, principalmente quando atingem os corpos de água, plantações, florestas e outros locais.

$$
\text { Nos Estados Unidos, o }
$$

Biodegradable Products Institute (BPI), que confere o selo de "biodegradável" aos materiais naquele país, não certifica os oxibiodegradáveis. Esta instituição chama este material de "oxodegradable", por considerarem que sua degradação ocorre exclusivamente pela oxidação, sem que haja a biodegradação (BPI, 2010).

No Brasil, contudo, a Associação Brasileira de Normas Técnicas (ABNT) certificou como oxibiodegradável 0 plástico aditivado com pró-degradantes (ex.: d2W $\mathrm{W}^{\mathrm{TM}}$ ), conforme a norma PE308.01 (ABNT, 2014). Daí o fato de os plásticos aditivados em nosso país serem tratados como oxibiodegradáveis.

0 presente trabalho foi conduzido com o objetivo de avaliar a degradação de embalagens plásticas biodegradáveis sob diferentes condições ambientais de descarte, no Município de São Lourenço do Oeste/SC, entre agosto e novembro de 2017.

\section{Material e métodos}

A pesquisa foi desenvolvida no Instituto Federal de Santa Catarina (IFSC), Campus São Lourenço do Oeste, localizado no Distrito de Frederico Wastner, Município de São Lourenço do Oeste, região oeste do Estado de Santa Catarina, tendo as seguintes coordenadas geográficas de referência $26^{\circ} 24^{\prime} 16^{\prime \prime} \mathrm{S}$ e 52.47' 5" W; com uma altitude aproximada de $800 \mathrm{~m}$. Segundo a classificação de Köppen (1900), o clima da região é caracterizado como $\mathrm{Cfb}$ (clima temperado úmido), com temperatura média anual oscilando em torno de $17{ }^{\circ} \mathrm{C}$ e precipitação média anual aproximada de $2.000 \mathrm{~mm}$. O período escolhido para o experimento foi entre os meses de agosto e novembro de 2017.

Como fator de estudo, foram testados cinco tratamentos simulando o descarte de sacolas plásticas produzidas em plástico oxibiodegradável de baixa densidade, com as medidas de $42 \times 53$ $\mathrm{cm}$, adquiridas no comércio local.

Os tratamentos foram os seguintes: $\mathrm{T} 1=\mathrm{a}$ céu aberto; $\mathrm{T} 2=$ abrigado; $\mathrm{T} 3=$ na água; $\mathrm{T} 4=$ sob solo seco; $\mathrm{T} 5=$ sob solo úmido. Cada tratamento constou de cinco replicações, sendo uma replicação representada por uma sacola plástica. Para a imposição dos tratamentos, com exceção do tratamento $\mathrm{T} 1$, as sacolas foram depositadas em bandejas plásticas com capacidade para 17 L (Figura 1). 

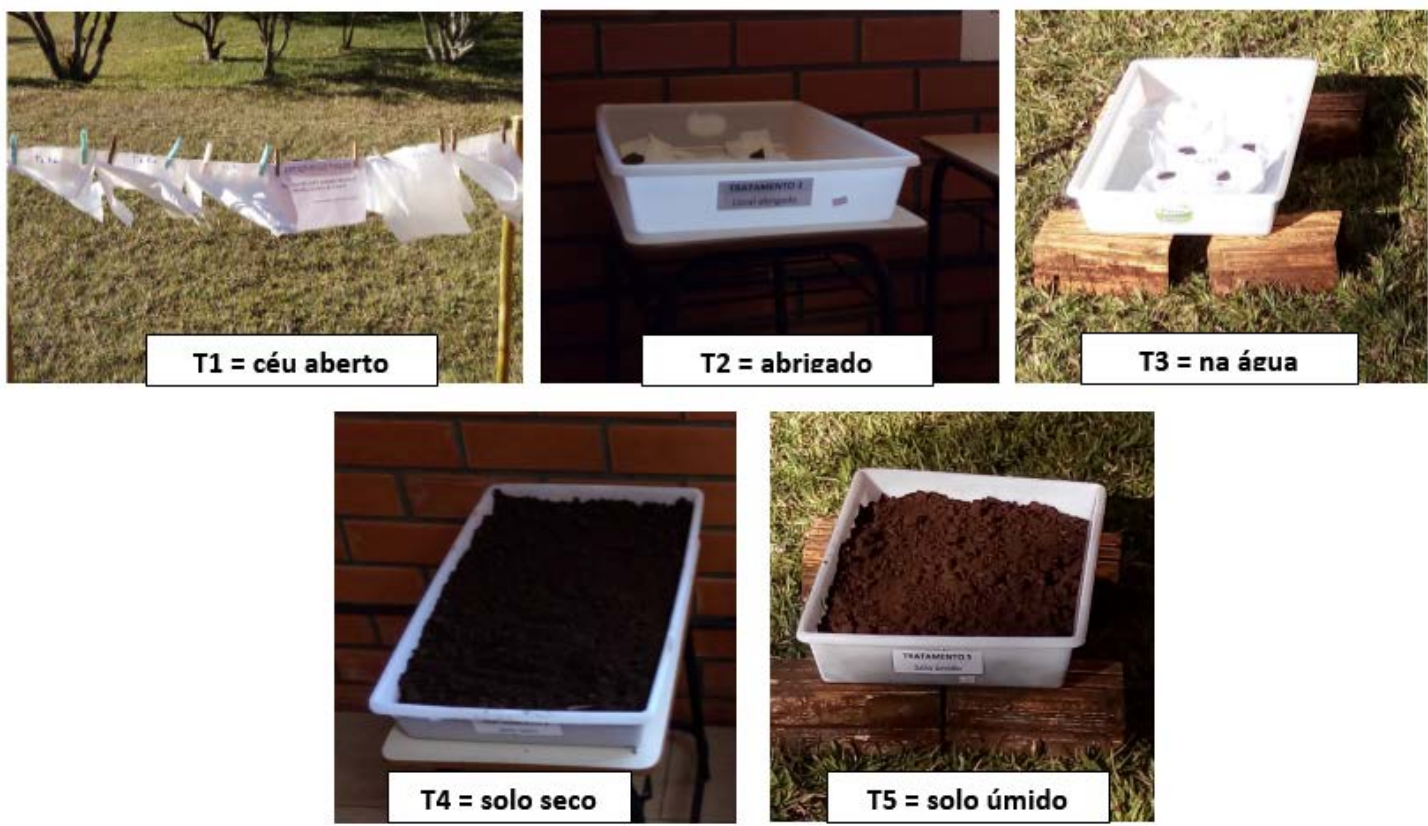

Figura 1. Tratamentos impostos às sacolas plásticas oxibiodegradáveis.

Os valores de temperatura e umidade relativa do ar foram monitoradas por meio de um termohigrômetro digital, em dois ambientes, interno (T2 e T4) e externo (T1, T3 e T5). Os valores médios obtidos $\left(\mathrm{T}{ }^{\circ} \mathrm{C} / \mathrm{UR} \%\right)$ no período foram: interno = $24{ }^{\circ} \mathrm{C} / 71 \%$; externo $=22^{\circ} \mathrm{C} / 78 \%$.

As coletas de dados foram realizadas aos 0, 30, 60 e 90 dias (d) de imposição dos tratamentos. A degradação das sacolas foi determinada mediante o decréscimo na massa (g) e aspectos visuais, comparando-se os períodos de exposição 30, 60 e 90 d com o período $0 \mathrm{~d}$.

As massas das sacolas foram determinadas em balança digital analítica 0,0001 x 220g, sendo os resultados para o decréscimo da massa expressos em: a) dados percentuais, obtidos por meio da fórmula: Decréscimo da massa $(\%)=\frac{(m i-m f)}{m i} \times 100$; em que: $\mathrm{m}_{\mathrm{i}}=$ massa inicial da sacola; $\mathrm{m}_{\mathrm{f}}=$ massa final da sacola; e b) comparação entre as médias obtidas nos tempos 0 e $90 \mathrm{~d}$. As médias foram comparadas via teste de média de Tukey ao nível de probabilidade de 5\%. Além da massa perdida em cada tratamento, foram obtidas fotos das sacolas em cada tempo de coleta a fim de expressar aspectos visuais da degradação das mesmas.

\section{Resultados e discussão}

0 principal parâmetro adotado para aferir a degradação das sacolas de filme plástico oxibiodegradável foi a perda de massa frente aos fatores ambientais impostos pelos tratamentos. A maior perda percentual de massa, após 90 dias, ocorreu no tratamento T1 (céu aberto), com um valor de 3,4\%, contrastando com a menor média, igual a $1,8 \%$, observada nas sacolas mantidas sob o abrigo do tempo (T2). Valores intermediários foram obtidos nos tratamentos T5 (solo úmido), T3 (na água) e T4 (solo seco), com respectivos 
percentuais de 3,13\%, 2,59\% e 1,94\% (Figura 2). Observando ainda a Figura $2 \mathrm{e}$ utilizando a equação da reta ajustada ao tratamento T5, aquele que apresentou os maiores dados de perda de massa, seriam necessários aproximadamente 332 dias para a decomposição total das sacolas.

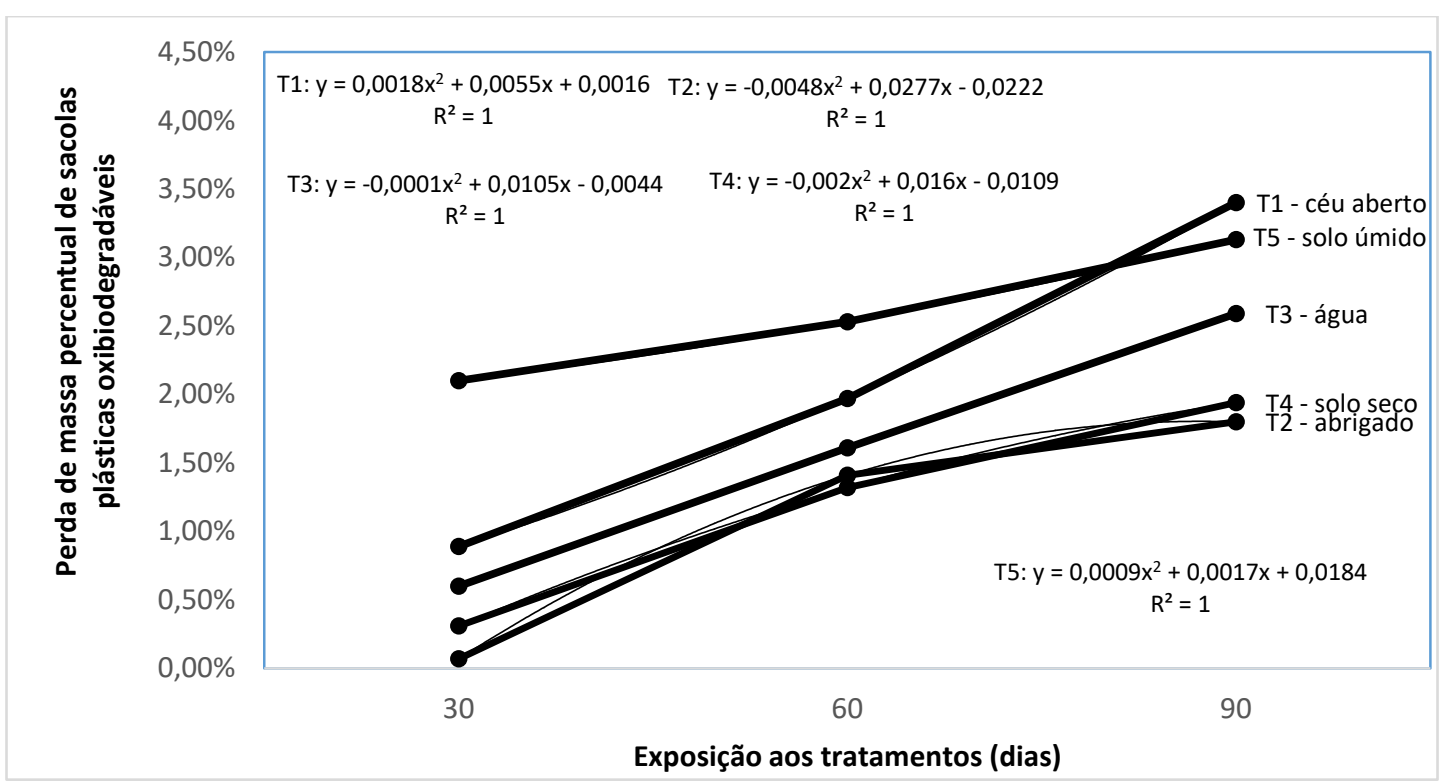

Figura 2. Decréscimo da massa (\%) de sacolas plásticas oxibiodegradáveis aos 30,60 e 90 dias expostas aos tratamentos T1 (ar livre); T2 (abrigado); T3 (água); T4 (solo seco) e T5 (solo úmido), em São Lourenço do Oeste/SC, de agosto a novembro de 2017.

A exposição aos tratamentos não promoveu alterações significativas na integridade física aparente das embalagens plásticas oxibiodegradáveis (Figura 3), ao término do experimento (90d), o que corrobora os resultados observados quanto à perda de massa observada (Figura 2), que expressou valores inferiores a $4 \%$ para quaisquer dos tratamentos testados.

Miranda et al. (2015), por meio de sua pesquisa científica, não obtiveram significativa degradação de sacolas plásticas oxibiodegradáveis e comuns em curtos períodos (até 90 dias), sob os efeitos da água da chuva e tratamentos de temperatura. No entanto, Follmann et al. (2017), investigando sacolas oxibiodegradáveis, obtiveram dados de degradação de perda de massa aproximada de $55 \%$, para um período de 3 meses, valores substancialmente superiores aos de nossa pesquisa.

As normas internacionais tratam não só apenas do tempo de degradação, mas do balanço de carbono dos materiais biodegradáveis, assim, a biodegradação deve ocorrer em até 180 dias e 60\% (Norma americana) e 90\% (Normas europeia e brasileira) do carbono contido no produto se transformem em $\mathrm{CO}_{2}$ (gás carbônico), água e um composto (Plastivida, 2008). Conforme Scott (2009) o plástico oxibiodegradável se destina a uma completa degradação em uma escala de tempo maior do que a compostagem (em torno de 180 dias), mas muito mais curto do que para os plásticos normais (10 anos ou mais). 


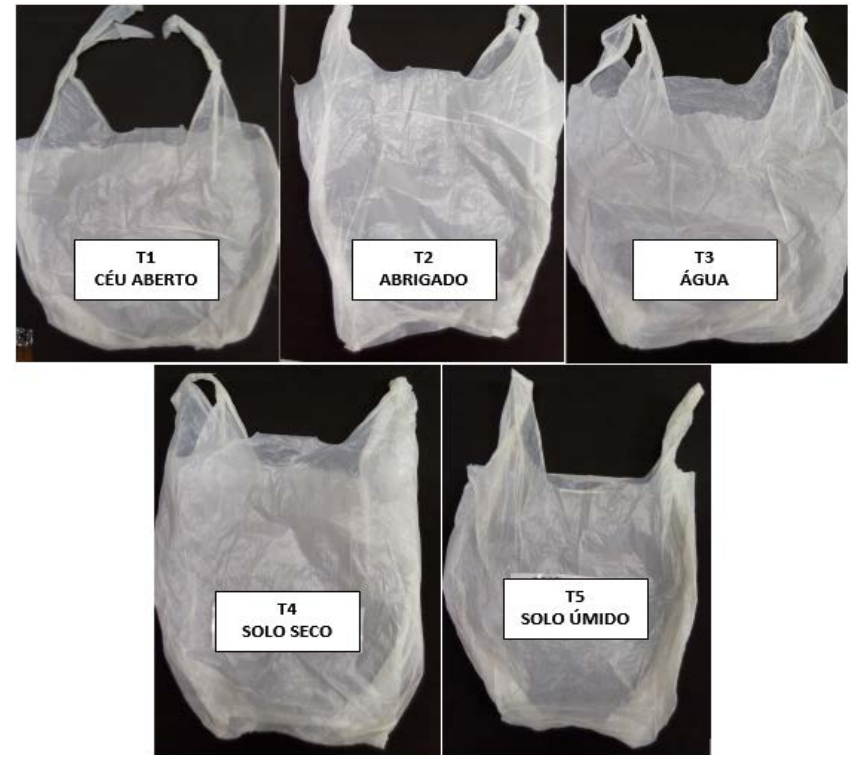

Figura 3. Aspecto visual das sacolas plásticas oxibiodegradáveis aos 90 dias de exposição aos tratamentos T1 (ar livre); T2 (abrigado); T3 (água); T4 (solo seco) e T5 (solo úmido), em São Lourenço do Oeste/SC, de agosto a novembro de 2017.

Além da perda de massa gradual, este trabalho comparou os valores médios das massas das sacolas, para cada tratamento, no início e o término do experimento. Estes resultados visaram a comparar a magnitude dos fatores degradantes (temperatura, luz, umidade e microrganismos) imposta em cada tratamento.

Quando comparadas as médias de massa nos diferentes tratamentos para os tempos 0 e 90 d, é possível observar que somente as formas de disposição das sacolas ao ar livre (T1), na água (T3) e em solo úmido (T5), promoveram perdas de massa significativas das embalagens plásticas oxibiodegradáveis. Assim, a diminuição da massa média das sacolas deixadas ao abrigo do tempo (T2) e enterradas em solo seco (T4) foi meramente visual, sem, portanto, alcançar valores estatisticamente diferentes (Tabela 1).

Tabela 1. Massa média de sacolas plásticas oxibiodegradáveis aos 0 e 90 dias de exposição aos tratamentos T1 (ar livre); T2 (abrigado); T3 (água); T4 (solo seco) e T5 (solo úmido), em São Lourenço do Oeste/SC, de agosto a novembro de 2017.

\begin{tabular}{|c|c|c|}
\hline Tratamento & Tempo de exposição (dias) & Massa média das sacolas (g)* \\
\hline \multirow{2}{*}{ T1 - ar livre } & 0 & $3,6880 \mathrm{a}$ \\
\hline & 90 & $3,5625 \mathrm{~b}$ \\
\hline \multirow{2}{*}{ T2 - abrigado } & 0 & $3,7042 \mathrm{a}$ \\
\hline & 90 & $3,6374 \mathrm{a}$ \\
\hline \multirow{2}{*}{ T3 - água } & 0 & $3,7035 \mathrm{a}$ \\
\hline & 90 & $3,6076 \mathrm{~b}$ \\
\hline \multirow{2}{*}{ T4 - solo seco } & 0 & 3,6838 a \\
\hline & 90 & $3,6122 \mathrm{a}$ \\
\hline \multirow{2}{*}{ T5 - solo úmido } & 0 & $3,7031 \mathrm{a}$ \\
\hline & 90 & $3,5873 \mathrm{~b}$ \\
\hline
\end{tabular}

*Médias seguidas por letras distintas diferem entre si ao nível de 5\% de probabilidade pelo teste de Tukey. 
Pode-se inferir que, para as condições do presente experimento, os tratamentos que mais promoveram a exposição das embalagens aos principais fatores degradantes (luz, umidade, temperatura e microrganismos), foram os que mais contribuíram para a degradação das sacolas plásticas oxibiodegradáveis. Caso do tratamento a céu aberto (T1), aquele que recebeu a maior incidência de luz solar e onde se constatou a maior perda de massa (percentual e absoluta) das embalagens.

Neste contexto, Manno et al. (2010) identificam a fotodegradação como a forma mais intensa de degradação de resíduos plásticos, pois está associada aos efeitos da radiação solar juntamente com a presença de oxigênio. Esse processo pode ocorrer pela ação conjunta de forças, como vento e chuva, fazendo com que ocorra uma diminuição do peso molecular e fissuras nas superfícies moldadas, tornando os plásticos quebradiços e esfarelados, gerando pequenos fragmentos. Entretanto, além de fatores abióticos como luz, umidade e temperatura, outra forma de degradação é a mediada por microrganismos ou biodegradação, em que os materiais se degradam em dióxido de carbono, água e biomassa, como resultado da ação de organismos vivos ou enzimas (Rosa et al., 2004).

Apesar de a biodegradabilidade dos plásticos com aditivo pró-degradante não ser aceita universalmente, Scott (2009) afirma que o plástico oxibiodegradável não se destina a se fragmentar somente, sendo concebido para uma completa bioassimilação por microrganismos naturais. Contudo, seria inconclusivo explicar o decréscimo estatisticamente significativo observado na massa das sacolas sob o tratamento solo úmido (Tabela 1), como resultado de ação microbiológica, o que demandaria um maior tempo de exposição ao tratamento. Pois a etapa de biodegradação só pode ocorrer posteriormente à quebra das moléculas do polímero via reação do oxigênio com o aditivo pró-degradante (Liplast, 2017).

\section{Conclusões}

Mediante os resultados obtidos, conclui-se que a degradação das sacolas oxibiodegradáveis atingiu valores de até $4 \%$ de perda de massa das embalagens.

Os tratamentos que simularam as condições de disposição das sacolas a céu aberto, na água e em solo úmido, foram os que promoveram decréscimos significativos na massa das embalagens, após 90 de exposição aos mesmos.

\section{Agradecimentos}

Os autores agradecem à Universidade Comunitária da Região de Chapecó - UNOCHAPECó, Campus São Lourenço do Oeste, pela gentileza em ceder as suas dependências para as determinações laboratoriais desta pesquisa.

\section{Declaração de conflito de interesses}

Os autores declaram não haver conflito de interesses.

\section{Referências}

ABNT - Associação Brasileira de Normas Técnicas. PE-308.01 Rótulo ecológico para aditivos plásticos com função oxibiodegradável. Rio de Janeiro: ABNT, 2014. Disponível em: <http://abnt.org.br/ criterios/app/arquivos/pdf/PE-

308.01_Rótulo Ecológico para Aditivos Plásticos com Função Oxibiodegradável.pdf>. Acesso em: 18 dez. 2017.

BPI - Biodegradable Products Institute. BPI position on degradable additives. 2010. Disponível em: <http://www.bpiworld.org/ BPI_Position_on_Degradable_Additives $>$.

Acesso em 19 dez. 2017.

Brasil. Lei no 12.305, de 2 de agosto de 2010. Institui a Política Nacional de Resíduos Sólidos; altera a Lei no 9.605, de 12 de fevereiro de 1998; e dá outras providências. Disponível em: <http://www.planalto. 
gov.br/ccivil_03/_ato2007-2010/2010/lei/ 112305.htm>. Acesso em: 18 dez. 2017.

Ecodebate. Sacola plástica é uma das maiores vilãs do meio ambiente. 2016. Disponível em: <https://www.ecodebate.com.br/2016/04/2 9/sacola-plastica-e-uma-das-maiores-vilasdo-meio-ambiente/>. Acesso em: $18 \mathrm{dez}$. 2017.

Follmann, A. J.; Rodrigues, A. C.; Camargo, M.; Follmann, D. N.; Souza, V. Q.; Graepin, C. Degradação de sacolas plásticas convencionais e oxibiodegradáveis. Ciência e Natura, v. 39, n. 1, p.187-192, 2017. https://doi.org/10.5902/2179460X24176

Köppen, W. Versuch einer Klassifikation der Klimate, vorzugsweise nach ihren Beziehungen zur Pflanzenwelt. (Schluss). Geographische Zeitschrift, v. 6, n. 12, p.657-679, 1900. Disponível em: <http://www.jstor.org/stable/27803939>. Acesso em: 18 dez. 2017.

Liplast. Entendendo melhor a oxibiodegradação do plástico. 2017. Disponível em: <http://www.liplast.com.br/blog/eventos/e ntendendo-melhor-a-oxibiodegradacao-doplastico>. Acesso em: 21 dez. 2017.

Manno, E. B.; Pacheco, E. B. A. V.; Bonelli, C. M. C. Meio ambiente, poluição e reciclagem. 2. ed. São Paulo: Blücher, 2010.

Miranda, C. S.; Seo, E. S. M. Degradação de embalagens plásticas oxibiodegradáveis e comuns. Holos Environment, v. 15, n. 2, p. 171-179, 2015. https://doi.org/10.14295/ holos.v15i2.10503

Oliveira, T. B.; Galvão Júnior, A. C. Planejamento municipal na gestão dos resíduos sólidos urbanos e na organização da coleta seletiva. Engenharia Sanitária e Ambiental, v. 21, n. 1, p. 55-64, 2016. https://doi.org/10.1590/S1413-41520201 600100155929

Pereira, S. S.; Curi, R. C. Modelos de gestão integrada dos resíduos sólidos urbanos: a importância dos catadores de materiais recicláveis no processo de gestão ambiental. In: Lira, W. S.; Cândido, G. A. (Orgs.). Gestão sustentável dos recursos naturais: uma abordagem participativa. Campina Grande: EDUEPB, 2013, p. 149-172.

Pinto, J. C.; Magrini, A.; Melo, C. K.; Castor Jr., C. A.; Gaioto, C. C.; Santos, D. P.; Borges, G.; Rosa, I. S.; Delgado, J. J. S.; Souza, M. N.; Oliveira, M. C. B. R.; Souza, P. N.; Melo Jr., P. A.; Aderne, R.; Vasconcelos, S. M. R. Impactos ambientais causados pelos plásticos: uma discussão abrangente sobre os mitos e os dados científicos. 1. ed. Rio de Janeiro: E-papers, 2012.

Plastivida. Mitos e fatos sobre plásticos oxidegradáveis. 2008. Disponível em: <http://www.plastivida.org.br/images/tema s/mitos-e-fatos- sobre-os-plasticos-oxidegradaveis.pdf>. Acesso em: 21 dez. 2017.

Polifilme Embalagens. 2013. Disponível em: <http://polifilme.com.br/index.php>. Acesso em: 15 dez. 2017.

Santos, A. S. F.; Freire, F. H. O.; Costa, B. L. N. Sacolas plásticas: destinações sustentáveis e alternativas de substituição. Polímeros, v. 22 , n. 3, p. 228-237, 2012. https://doi.org/ 10.1590/S0104-14282012005000036

Rosa, D. S.; Lotto, N. T.; Guedes, C. G. F. The use of roughness for evaluating the biodegradation of poly- $\beta$-(hydroxybutyrate) and poly- $\beta$-(hydroxybutyrate-co- $\beta$-valerate). Polymer Testing, v. 23, n. 3, p. 3-8, 2004. https://doi.org/10.1016/S0142-9418(03) 00042-4

Scott, G. Oxibiodegradable plastic. Bioplastics Magazine, v. 4, p 28-30, 2009. Disponível em: <http://www.bioplastics magazine.com/bioplasticsmagazinewAssets/docs/article/0905_p30_bioplastics MAGAZINE.pdf>. Acesso em: 15 dez. 2017. 